\title{
Electromagnetic source imaging in presurgical workup of patients with epilepsy
}

\author{
A prospective study
}

Lene Duez, MD, PhD, Hatice Tankisi, MD, PhD, Peter Orm Hansen, MD, PhD, Per Sidenius, MD, DMSc, Anne Sabers, MD, DMSc, Lars H. Pinborg, MD, DMSc, Martin Fabricius, MD, DMSc, György Rásonyi, MD, Guido Rubboli, MD, DMSc, Birthe Pedersen, MD, Anne-Mette Leffers, MD, Peter Uldall, MD, DMSc, Bo Jespersen, MD, Jannick Brennum, MD, DMSc, Otto Mølby Henriksen, MD, PhD, Anders Fuglsang-Frederiksen, MD, DMSc, ${ }^{*}$ and Sándor Beniczky, MD, PhD*

Neurology ${ }^{\circledR}$ 2019;92:e576-e586. doi:10.1212/WNL.0000000000006877

\begin{abstract}
Objective

To determine the diagnostic accuracy and clinical utility of electromagnetic source imaging (EMSI) in presurgical evaluation of patients with epilepsy.
\end{abstract}

\section{Methods}

We prospectively recorded magnetoencephalography (MEG) simultaneously with EEG and performed EMSI, comprising electric source imaging, magnetic source imaging, and analysis of combined MEG-EEG datasets, using 2 different software packages. As reference standard for irritative zone (IZ) and seizure onset zone (SOZ), we used intracranial recordings and for localization accuracy, outcome 1 year after operation.

\section{Results}

We included 141 consecutive patients. EMSI showed localized epileptiform discharges in 94 patients (67\%). Most of the epileptiform discharge clusters (72\%) were identified by both modalities, $15 \%$ only by EEG, and $14 \%$ only by MEG. Agreement was substantial between inverse solutions and moderate between software packages. EMSI provided new information that changed the management plan in 34\% of the patients, and these changes were useful in $80 \%$. Depending on the method, EMSI had a concordance of $53 \%$ to $89 \%$ with IZ and $35 \%$ to $73 \%$ with SOZ. Localization accuracy of EMSI was between $44 \%$ and $57 \%$, which was not significantly different from MRI (49\%-76\%) and PET (54\%-85\%). Combined EMSI achieved significantly higher odds ratio compared to electric source imaging and magnetic source imaging.

\section{Conclusion}

EMSI has accuracy similar to established imaging methods and provides clinically useful, new information in $34 \%$ of the patients.

\section{Classification of evidence}

This study provides Class IV evidence that EMSI had a concordance of 53\%-89\% and $35 \%-73 \%$ (depending on analysis) for the localization of epileptic focus as compared with intracranial recordings- $-\mathrm{IZ}$ and SOZ, respectively.

\author{
Correspondence \\ Prof. Beniczky \\ sandor.beniczky@ \\ aarhus.rm.dk
}

- CME Course

NPub.org/cmelist

\section{$\rightarrow$ Class of Evidence \\ Criteria for rating therapeutic and diagnostic studies \\ NPub.org/coe}

\footnotetext{
*These authors contributed equally to this work.

From the Departments of Clinical Neurophysiology (L.D., H.T., P.O.H., A.F.-F., S.B.) and Neurology (P.S.), Aarhus University Hospital; Departments of Neurology (A.S., L.H.P.), Clinical Neurophysiology (M.F., G. Rásonyi), Pediatrics, Child Neurology (P.U.), Neurosurgery (B.J., J.B.), and Clinical Physiology, Nuclear Medicine and PET (O.M.H.), Copenhagen University Hospital Rigshospitalet; Danish Epilepsy Centre (G. Rubboli, B.P., S.B.), Dianalund; and Department of Diagnostic Radiology (A.-M.L.), Hvidovre Hospital, Denmark.

Go to Neurology.org/N for full disclosures. Funding information and disclosures deemed relevant by the authors, if any, are provided at the end of the article. 


\section{Glossary}

BESA = Brain Electrical Source Analysis; cEMSI = combined electromagnetic source imaging; DSM = distributed source model; ECD = equivalent current dipole; ED = epileptiform discharge; EMSI = electromagnetic source imaging; ESI = EEG source imaging; FDG $={ }^{18}$ F-fluorodeoxyglucose; FLAIR = fluid-attenuated inversion recovery; FN = false negative; FP = false positive; GLM = generalized linear model; ICR = intracranial recording; IZ = irritative zone; $\mathbf{M D T}=$ multidisciplinary team; MEG = magnetoencephalography; MPRAGE = magnetization-prepared rapid-acquisition gradient echo; MSI = magnetoencephalography source imaging; NPV = negative predictive value; $\mathbf{O R}=$ odds ratio; $P P V=$ positive predictive value; $\mathrm{SOZ}=$ seizure onset zone; $\mathbf{T N}=$ true negative; $\mathbf{T P}=$ true positive.

Epilepsy has a prevalence of 4-10/1,000 persons, and up to $30 \%$ do not respond to antiepileptic drugs. ${ }^{1}$ Surgery is a therapeutic option for patients with drug-resistant focal epilepsy. ${ }^{2}$ Depending on the ability to localize and resect the epileptogenic zone, $30 \%$ to $85 \%$ of operated patients become seizure-free. ${ }^{3}$

The greatest challenge in epilepsy surgery is the presurgical evaluation. On their own, none of the methods can reliably identify the epileptogenic zone. Therefore, a multimodal approach using semiology, EEG, and MRI is needed. These are often supplemented with functional neuroimaging methods, such as SPECT or PET. ${ }^{4}$ In difficult cases, intracranial electrodes are implanted. ${ }^{3}$

Advances in recording techniques and in signal analysis have made it possible to estimate the source of the epileptiform discharges (EDs) recorded by EEG (EEG source imaging: ESI) and magnetoencephalography (MEG source imaging: MSI). ${ }^{4}$ In studies ${ }^{5,6}$ investigating the clinical utility of MSI (i.e., changes in clinical decision-making, based on MSI), MSI led to a change in the management plan in $21 \%$ to $33 \%$ of patients, and this was associated with long-term seizure control. Large prospective studies showed that ESI accurately estimated the location of the epileptic focus, ${ }^{7,8}$ although its role in clinical decision-making was not addressed in those studies.

It is possible to record MEG and EEG signals simultaneously, and perform electromagnetic source imaging (EMSI), analyzing both modalities either separately (ESI and MSI) or combined (cEMSI). Despite numerous studies suggesting that these methods are accurate, ESI and MSI are only implemented in a few centers, ${ }^{5}$ and often EEG and MEG are not recorded simultaneously. This is probably attributable to the lack of welldesigned, large, prospective, blinded studies addressing EMSI. Many questions remain unanswered: What is the diagnostic yield of simultaneous MEG and EEG recordings? Which inverse solution is best? Do different software packages and operators give similar results? Can the methods accurately estimate the source of the EDs? Do they provide nonredundant, clinically useful information that changes and improves decision-making in presurgical evaluation?

To elucidate the role of EMSI in presurgical evaluation, we conducted a prospective, blinded study. We evaluated the diagnostic yield of the 2 recorded modalities, inter-software and -operator agreement, accuracy of source localization, and its clinical utility. We designed the study and we reported it according to the STARD (Standards for Reporting Diagnostic Accuracy) criteria. ${ }^{9}$

\section{Methods}

\section{Study design and patients}

From April 2011 to June 2016, we prospectively recruited consecutive patients undergoing presurgical evaluation, as part of the Danish Epilepsy Surgery Program. All patients had drug-resistant focal epilepsy, and for all patients, at least 2 , appropriately chosen and taken antiepileptic drugs had failed. The intended sample size was $\geq 100$ patients, based on previous studies. ${ }^{10}$ This number has proven to provide the reference standard in $>50$ patients undergoing intracranial recording (ICR) or operation. ${ }^{11}$

Inclusion criteria were patients who underwent presurgical evaluation and who gave their consent. There were no exclusion criteria; data from all recruited patients were analyzed.

\section{Standard protocol approvals, registrations, and patient consents}

The study was approved by the regional ethics committee (case number 1-10-72-177-12) and patients gave their informed written consent. Patients underwent presurgical evaluation according to the investigation protocol approved by the Danish Health Authority (data available from Dryad, appendix 1, doi.org/10.5061/dryad.p4r01pq). This included detailed history, semiology, and EEG from video-EEG monitoring, MRI, and neuropsychological tests for all patients. When decisions could not be made based on these data, patients were referred to additional noninvasive investigations (PET, EMSI) and ICRs (stereo-EEG).

Presurgical patients, in whom the multidisciplinary team (MDT) reached a conclusion based on semiology, EEG, and MRI, were recruited as part of the research study $(\operatorname{arm} A)$. In these patients, EMSI was not part of the decision-making. Patients with normal MRI or discordant data (semiology, EEG, MRI) were referred to PET and EMSI as part of their presurgical evaluation, before ICR ( $\operatorname{arm} B)$. In these patients, EMSI was part of the decision-making process. 
For patients in arm B, the MDT had a 2-step decision process. Decision was first made blinded to EMSI, based on all other data, and it was logged into the database. Decisions were categorized as (1) stop (operation not offered), (2) implantation of intracranial electrodes (specifying their location), or (3) operation (resective surgery). Then, a second decision was made, based on new information (if any) provided by the EMSI, weighted against all other data. Possible changes in the management plan included any change from one of these categories to another and change within category 2 (i.e., change in the planned intracranial electrode positions).

\section{Simultaneous MEG and EEG recordings}

MEG-EEG was recorded at Aarhus University Hospital, using an Elekta Neuromag system (Elekta, Stockholm, Sweden).

MEG was recorded at 102 evenly distributed locations, with 3 sensors at each location ( 2 orthogonal planar gradiometers and a magnetometer), giving a total of 306 channels. Continuous head position indicators were used. MEG data were preprocessed offline using the spatiotemporal signal space separation method ${ }^{12}$ to suppress the residual interference and to correct for head movements.

High-density EEG (64-80 electrodes) was recorded using a nonmagnetic cap (EASYCAP) and additional electrodes covering the inferior part of the head (cheek, inferior temporal chain, and neck) in all patients with head circumference $<60$ $\mathrm{cm}$ and who could cooperate with the procedure. In other patients, an array of 19 electrodes (10-20 system) or no EEG was recorded. In addition, electrooculogram and ECG were recorded. Electronic tracking of positions of electrodes and of the head position coils were done using a Polhemus system (Polhemus, Colchester, VT). ${ }^{13}$

Spontaneous activity (eyes closed, rest, supine position) was recorded for 60 to 90 minutes (sampling frequency $1 \mathrm{kHz}$; online bandpass $0.1-330 \mathrm{~Hz}$ ) both for MEG and EEG. Data were offline bandpass filtered at 0.5 to $70 \mathrm{~Hz}$.

\section{Electromagnetic source imaging}

MEG-EEG was inspected for EDs by 2 trained experts (L.D., S.B.). Signals were analyzed using 2 commercially available software packages, with European approval for medical use (CE-mark). L.D. used the CURRY 7 Neuroimaging Suite (Compumedics, Victoria, Australia). S.B. used BESAResearch 6 (BESA, Gräfelfing, Germany). Voltage maps and magnetic field maps were inspected, and EDs were grouped in different clusters when the topographic maps differed. Then, for each cluster, using the visually detected EDs as templates, automated algorithms (template matching) scanned the recordings, and detected EDs were visually checked. To improve the signal-to-noise ratio, EDs with similar topography were averaged. ${ }^{14}$

Sequential topographic maps were inspected in the time epoch of the ascending slope of the averaged waveforms for change in topography indicating propagation. In addition, principal components analysis was used to identify propagation. ${ }^{15}$ When propagation occurred, source imaging was done first at the onset epoch, and then at the peak. When propagation was not identified, source imaging was done at the middle third of the ascending slope. ${ }^{10}$ With both software packages, 2 different inverse solutions were applied: equivalent current dipole (ECD) and a distributed source model (DSM): sLORETA, ${ }^{16}$ with an anatomically constrained linear estimation approach, assuming the sources were distributed in the cerebral cortex in CURRY, and CLARA in BESA. ${ }^{8}$ ESI and MSI were done with both software packages. In addition, using CURRY, source modeling of both datasets (EEGMEG) was done (cEMSI). ${ }^{17}$

All analyses were performed using individual head models from the patients' MRIs, coregistered with the MEG-EEG recording using fiducial landmark positions, electrode positions, and head position coils, digitized before the recording. In CURRY, 3-compartment boundary element method (BEM) head model was used. ${ }^{18}$ In BESA, an individual 4-layer finite element method (FEM) head model was used. ${ }^{14}$ Figure 1 illustrates the steps of the EMSI.

EMSI was performed prospectively, blinded to clinical information and reference standard results.

\section{Conventional neuroimaging methods}

MRI and ${ }^{18}$ F-fluorodeoxyglucose (FDG)-PET were performed as part of the presurgical evaluation. The MRI epilepsy protocol consisted until 2015 of a sagittal T1 3-dimensional (3D) magnetization-prepared rapid-acquisition gradient echo (MPRAGE) sequence, coronal and axial fluidattenuated inversion recovery (FLAIR) sequences, and a coronal T2-weighted (W) fast spin-echo sequence. Coronal and axial FLAIR and T2W sequences were performed perpendicular to and in plane with hippocampus long-axis. Since 2015, the protocol consisted of sagittal T1 3D MPRAGE sequence, sagittal 3D FLAIR sequence, coronal and axial T2W sequences, perpendicular and horizontal to hippocampus long-axis, axial susceptibility-weighted image sequence, axial diffusion-weighted image sequence, sagittal T1 3D MPRAGE sequence, and axial T1W spin-echo sequence after IV contrast. All 3D sequences were reconstructed in coronal and axial planes perpendicular and horizontal to the hippocampus long-axis.

For PET, FDG was administered IV with the patient in supine position and equipped with eye pads and earplugs. PET scan was commenced 40 minutes after tracer injection. PET scans were coregistered and displayed superimposed on MRI sequences using a standard clinical workstation and dedicated software supplied by the camera vendor (Leonardo station using TrueD; and from 2013 syngo.via using MI Neurology, Siemens Medical Solutions, Malvern, PA) who also provided access to age-matched normal database comparison. 


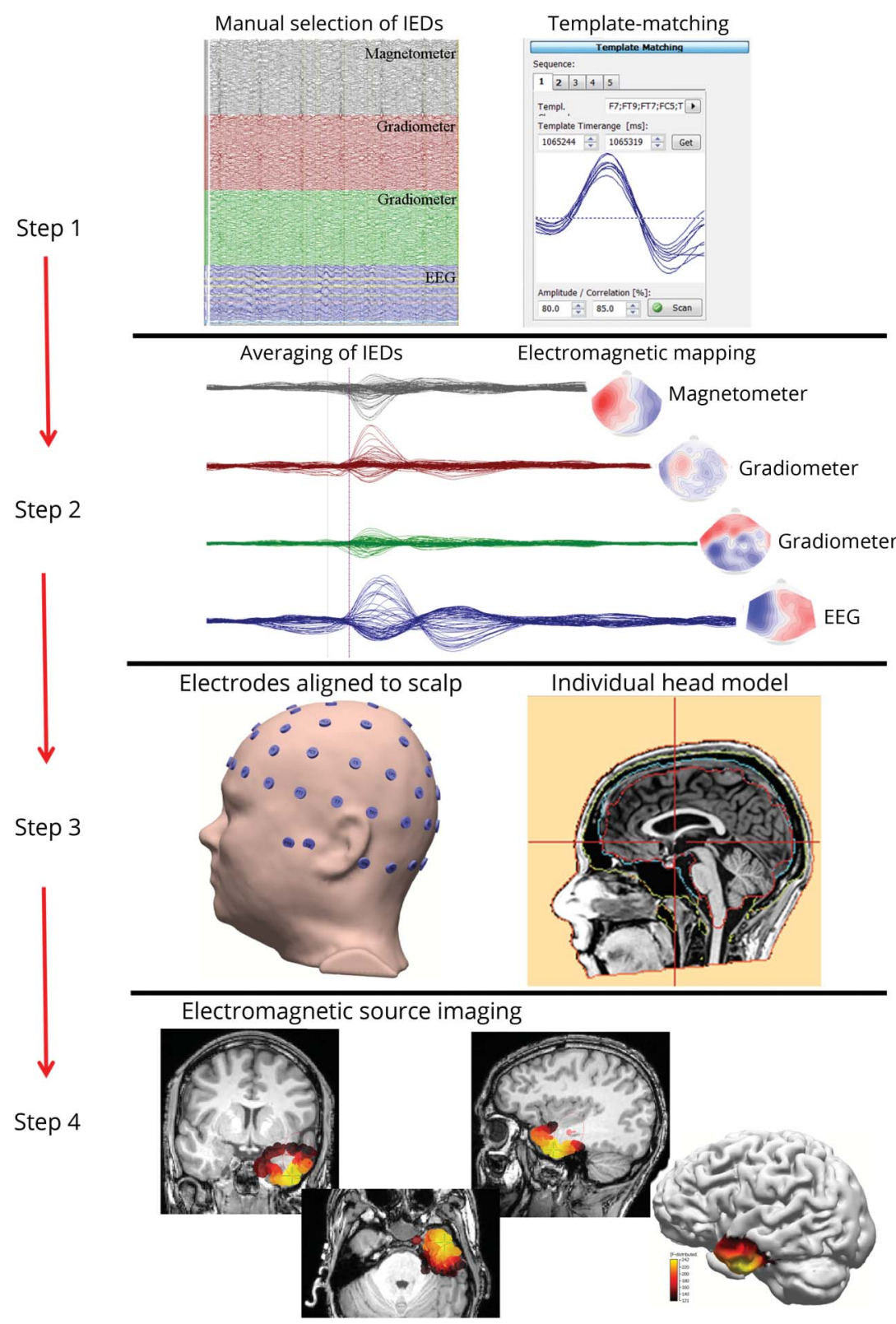

EMSI was performed using the following 4 steps: (1) review of the magnetoencephalography and EEG recordings and visual identification and marking of EDs belonging to the same cluster. These EDs were used for template-matching. Each detected spike was visually checked and artifacts were discarded. (2) EDs within each cluster were averaged to improve signalto-noise ratio (critical for spike onset activity relative to the background activity). ${ }^{28}$ Sequential topographic plots of the ascending phase and principal components analysis was used to identify propagation. (3) Individual head model was created for each patient, and the EEG electrodes were aligned to the scalp. (4) Source modeling was performed using 2 different inverse-solution strategies: equivalent current dipole and distributed source models where yellow indicates maximum intensity. $E D=$ epileptiform discharge; $\mathrm{EMSI}$ = electromagnetic source imaging; IED = interictal epileptiform discharge.

\section{Sublobar brain regions}

EMSI, PET, and MRI localization results were prospectively logged into the database, with localization scores at sublobar level, corresponding to 64 categories. This included the sublobar regions proposed by an international consensus paper and IFCN (International Federation of Clinical Neurophysiology) guideline. ${ }^{15,19}$ A descriptor of the side (left/right/mesial) was added. When more than 2 independent foci were identified, and none of them had a dominant activity, the result was scored as multifocal. A focus was considered dominant if the number of EDs was more than twice the number of EDs from all other foci. The remaining categories were (1) normal and (2) not localizable. Not localizable was when the ECD confidence interval or the distributed source analysis spanned over more than one lobe.

\section{Reference standard}

Ideal reference standard lacks for source imaging in presurgical evaluation. ${ }^{6}$ Accurately localizing the generator of interictal EDs (the irritative zone [IZ]) does not necessarily imply that resection of that area will render the patient seizure-free. Therefore, we used 2 different sets of reference standards: ICR and outcome after surgery (see below).

\section{Outcome measures}

First, the number of ED clusters recorded by only MEG and only EEG were compared. 
Agreement between different EMSI methods was calculated. All comparisons were done at sublobar level.

Clinical utility of EMSI was defined as the proportion of patients in whom EMSI changed the decision of the MDT. A change was defined useful as follows: (1) change from stop to ICR - the ICR localized the source; (2) change in implantation strategy - the electrode(s) implanted based on the EMSI identified the source; (3) change from implantation to operation without preceding implantation - the patient became seizure-free (at 1-year postoperative follow-up).

For EMSI, PET and MRI, we calculated the following outcome measures. Agreement with ICR: concordance with IZ and concordance with the seizure onset zone (SOZ) were determined using ICR as reference standard. Proportion of patients with results concordant with the reference standard, and agreement with this reference standard were calculated.

Sensitivity, specificity, localization accuracy, positive predictive value (PPV), and negative predictive value (NPV) were determined using outcome 1 year after surgery as reference standard. Preoperative EMSI was coregistered with the postoperative MRI; the source was considered concordant with the resected site when the ECD and respectively the maximum of the DSM was within the operation cavity. All other results (localization outside the cavity, multifocal EDs, normal recoding, not-localizable results) were considered discordant. We categorized the localizations as follows: TP (true positive) — concordant with the resection and seizurefree; FP (false positive) - concordant with the resection and not seizure-free; TN (true negative)—discordant with the resection and not seizure-free; FN (false negative)discordant with the resection and seizure-free. We used the following formula:

$$
\begin{gathered}
\text { Sensitivity }=\mathrm{TP} /(\mathrm{TP}+\mathrm{FN}) \\
\text { Specificity }=\mathrm{TN} /(\mathrm{TN}+\mathrm{FP}) \\
\text { Accuracy }=(\mathrm{TN}+\mathrm{TP}) /(\mathrm{TP}+\mathrm{FP}+\mathrm{TN}+\mathrm{FN}) \\
\mathrm{PPV}=\mathrm{TP} /(\mathrm{TP}+\mathrm{FP}) \\
\mathrm{NPV}=\mathrm{TN} /(\mathrm{TN}+\mathrm{FN})
\end{gathered}
$$

Surgical procedures were tailored to each patient, based on the final conclusion of the MDT.

We calculated the odds ratio (OR) of becoming seizure-free when operation was concordant vs discordant with the localization of the index test: $\mathrm{OR}=(\mathrm{TP} / \mathrm{FP}) /(\mathrm{FN} / \mathrm{TN})$.

\section{Statistics}

For assessment of agreement $(\kappa)$ between EMSI methods, software packages, and operators, and for assessment of agreement between EMSI and ICR, we calculated Gwet $\mathrm{AC}_{1}{ }^{20}{ }^{20}$ We used this measure of agreement because it yields more reasonable values than the Cohen $\kappa$ in case of low-trait prevalences. ${ }^{20}$ Interrater agreement was interpreted according to the conventional groups: poor $(\kappa<0)$, slight $(\kappa: 0.01-0.2)$, fair ( $\kappa: 0.21-0.4)$, moderate ( $\kappa: 0.41-0.6)$, substantial ( $\kappa$ : $0.61-0.8)$, and almost perfect agreement $(\kappa>0.8){ }^{21}$

The effect measures of sensitivity, specificity, PPV, NPV, concordance with SOZ, localization accuracy, and their 95\% confidence intervals were estimated using a generalized linear model (GLM) ${ }^{22,23}$ Identity link function was used in the GLM to compare index tests by means of the differences of the effect measure. Multiple observations per patient were considered in the GLM by specifying the patient ID as a cluster. Analysis was performed in StataCorp software release 14 (StataCorp LP, College Station, TX) using the binreg function.

\section{Classification of evidence}

The study was designed to answer the following question: What is the localization accuracy and clinical utility of EMSI in presurgical evaluation of patients with drug-resistant focal epilepsy?

The study was prospective and blinded. All patients who underwent presurgical evaluation and who gave their informed consent were recruited. Data from all recruited patients were analyzed and evaluated. All patients had drugresistant focal epilepsy.

This study provides Class IV evidence that EMSI had a concordance of $53 \%-89 \%$ and $35 \%-73 \%$ (depending on analysis) for the localization of epilepsy as compared with ICRs-IZ and SOZ, respectively.

\section{Data sharing}

Individual deidentified data, including localization results of the imaging methods, reference standard localizations, and outcome data will be shared, along with the following related documents: study protocol and statistical analysis plan. Unrestricted access to these data will be made available from the day of the online publication of the article, until 2030, using a publicly accessible repository (datadryad.org//) (Dryad doi:10.5061/dryad.p4r01pq). During the review phase, the data package is temporarily available via the following link: datadryad.org/review?doi=doi:10.5061/dryad.p4r01pq.

\section{Results}

Figure 2 shows examples of EMSI. Source images coregistered with postoperative MRIs are shown in data available from Dryad (appendix 2, doi.org/10.5061/dryad.p4r01pq). Figure 3 shows the flowchart of presurgical evaluation of all recruited patients.

\section{Patients}

One hundred forty-one consecutive patients (59 females) were included. Median age was 32 years (range: 8-70). All 

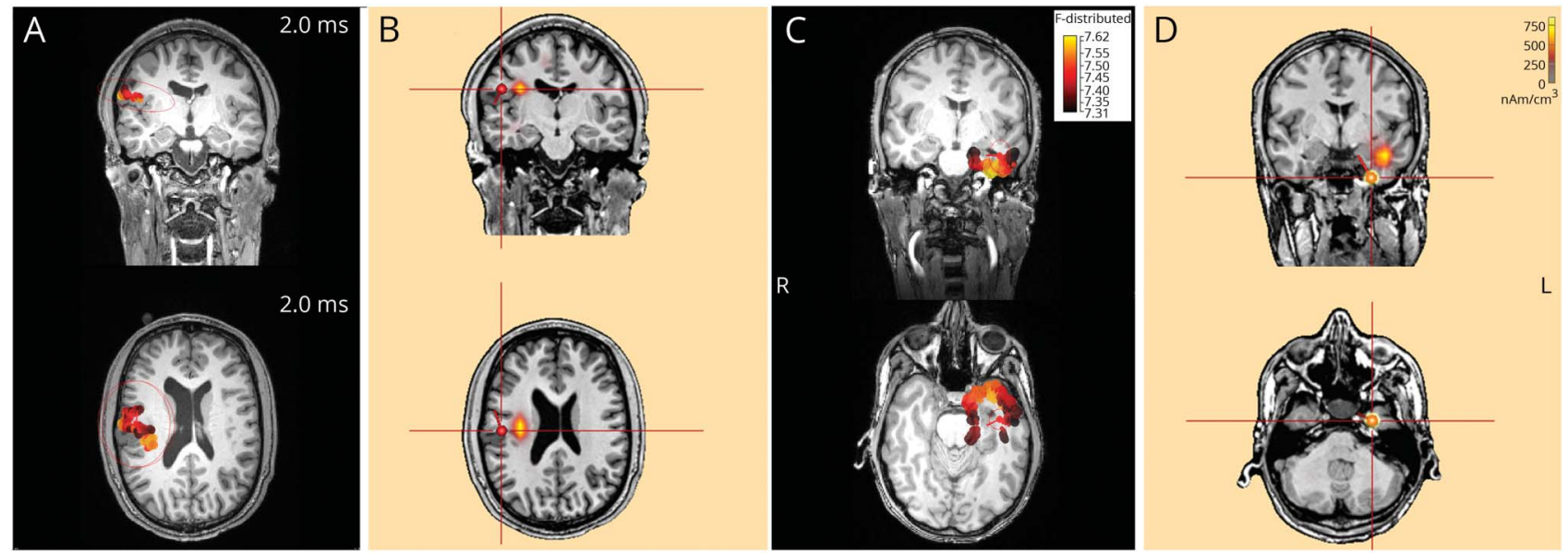

Electromagnetic source imaging (equivalent current dipole and distributed source model) for a patient with frontal ( $A$ and $B)$ and temporal (C and $D)$ focus. Analysis was done using CURRY ( $A$ and $C$ ) and BESA ( $B$ and D) software. Figures in appendix 2 (data available from Dryad, doi.org/10.5061/dryad.p4r01pq) show preoperative sources coregistered with postoperative MRI for these patients.

patients had MRI and MEG. Simultaneously with MEG, 115 patients had high-density EEG (64-80 electrodes). Because of large head circumference or inability to cooperate, 8 patients had an array of 19 EEG electrodes and 18 patients did not have EEG. One hundred ten patients had PET. MRI showed potentially epileptogenic lesions in 68 patients (48\%). PET showed a functional deficit zone in 70 patients (63\%). Seventy-two patients had temporal foci and 66 had extratemporal foci (frontal 50, parietal 11, occipital 5). Three patients could not be localized. The time from MEG-EEG recording to operation ranged from 1 to 48 months. No adverse events occurred from performing MEG-EEG.

\section{Detection of EDs by EEG and MEG}

EMSI showed focus localized to one or more sublobar regions in 94 patients (67\%). The remaining patients had normal MEG-EEG (46 patients; 33\%) or the source was not localizable (one patient). See data available from Dryad, appendices 3 and 4, doi.org/10.5061/dryad.p4r01pq.

In patients who had simultaneous MEG and EEG recordings $(\mathrm{n}=123)$, a total of 96 clusters of EDs were identified in 85 patients (60\% of all patients). In $72 \%(70 / 96)$, EDs were visible both in MEG and EEG. In 15\% (14/96), EDs were visible only in EEG. In 13\% (12/96), EDs were visible only in MEG. There was no significant difference in the number of interictal foci detected only by EEG and only by MEG ( $p=$ 0.67 ). Of the 14 foci detected only by EEG, 9 were located in the temporal lobe. Of the 12 foci detected only by MEG, 7 were temporal.

\section{Agreement between EMSI methods}

Between-software and -operator agreement: both for ECD and for DSM, agreement between BESA and CURRY was moderate, for both modalities (ESI or MSI) (table 1).
Agreement in localization between the 2 inverse solution strategies (ECD and DSM) within the same software and operator: using BESA, there was almost perfect agreement for MSI and substantial agreement for ESI (table 1). When using CURRY, there was substantial agreement between ECD and DSM for both modalities (ESI and MSI) (table 1).

\section{Clinical utility of EMSI}

The effect of EMSI on patient management planning was assessed in 85 patients (50 males; age: 10-70, median 32 years) in whom EMSI was part of the decision-making process. In this subgroup, 38 patients were MRI-negative; in the remaining patients, there were discordances between MRI and data from long-term video-EEG monitoring (semiology and EEG).

In 34\% (29/85), EMSI changed the management plan. Figure 4 shows the types of changes in the management plan, based on EMSI. At 1-year follow-up, reference standard was available for 20 patients ( 5 patients did not wish to proceed with ICR, one patient withdrew before operation, and 3 patients were still on the waiting list). In $80 \%(16 / 20)$, these changes proved to be useful: in $4 / 4$ patients who proceeded to implantation based on EMSI, IZ and SOZ had been identified; in 8/10 patients in whom additional electrodes were implanted, the additional electrodes identified IZ and SOZ; 4/6 patients who skipped implantation and proceeded to operation became seizure-free. See data available from Dryad, appendix 5, doi.org/10.5061/dryad.p4r01pq.

\section{Concordance with ICR}

Fifty-three patients (34 males; age: 8-60, median 40 years) underwent ICR; 25 were MRI-negative. Twenty-nine patients had temporal foci and 24 had extratemporal foci (frontal 16, 
Figure 3 Flowchart of the presurgical evaluation for the 141 recruited patients

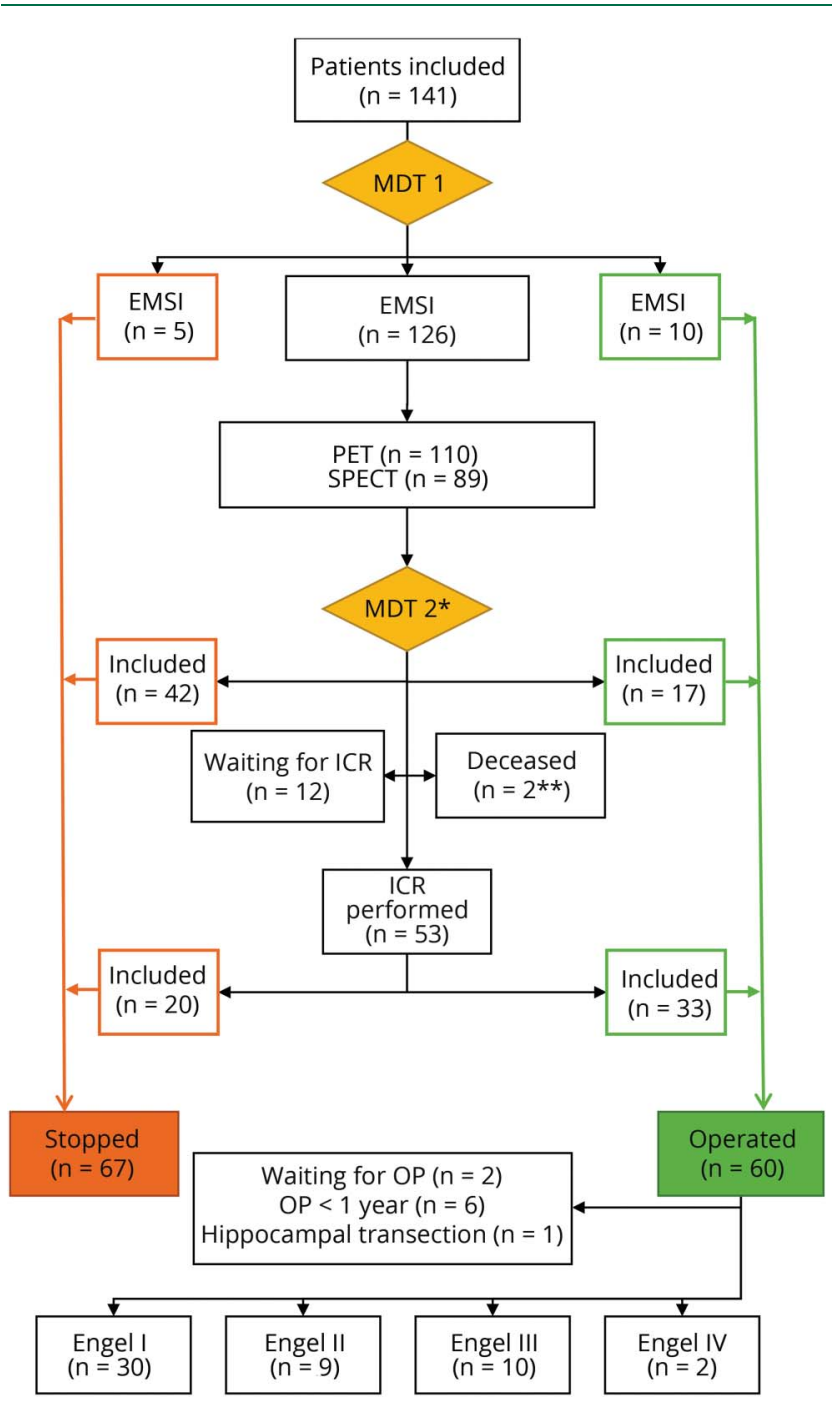

Red arrows and boxes indicate that operation was not offered and green arrows and boxes indicate that operation was indicated, by the MDT. *At this stage in the flowchart, the MDT made 2-step decisions: first blinded to EMSI, then including EMSI results. **One patient died of acute myocardial infarction and one patient died of sudden unexpected death in epilepsy. EMSI = electromagnetic source imaging; ICR = intracranial recording; MDT = multidisciplinary team; OP = operation

parietal 6, occipital 2). SOZ could not be localized by ICR in 14 of 53 patients.

Concordance between EMSI and the IZ was between 53\% and $89 \%$ (table 2). For ECD, this was higher in BESA than in CURRY, both for EEG $(p=0.0003)$ and MEG $(p=0.0107)$; for DSM, there was no significant difference between the 2 software packages. Concordance with IZ was higher for most of the EMSI methods than for MRI (50\%; 31\%-69\%) and for PET (29\%; 13\%-45\%) (data available from Dryad, appendices 6 and 7, doi.org/10.5061/dryad.p4r01pq).

Agreement between BESA EMSI and IZ was substantial. Agreement between CURRY MEG DSM and IZ was
Table 1 Agreement between electromagnetic source imaging methods

\begin{tabular}{lll}
\hline \multicolumn{1}{l}{ MEG } & EEG \\
\hline BESA vs CURRY & & \\
\hline ECD & $0.58(0.48-0.69)$ & $0.47(0.34-0.58)$ \\
\hline DSM & $0.58(0.49-0.68)$ & $0.43(0.33-0.54)$ \\
\hline BESA & & \\
\hline ECD vs DSM & $0.85(0.78-0.91)$ & $0.72(0.63-0.82)$ \\
\hline CURRY & & \\
\hline ECD vs DSM & $0.71(0.62-0.80)$ & $0.63(0.52-0.73)$ \\
\hline
\end{tabular}

Abbreviations: DSM = distributed source model; ECD = equivalent current dipole; $\mathrm{MEG}=$ magnetoencephalography.

Between-software and -operator agreement (BESA vs CURRY) and agreement between inverse solution strategies (ECD vs DSM) within the same software and operator. Data represent $\mathrm{k}$ values (confidence intervals).

substantial; for the other CURRY EMSI, it was moderate (table 3). Agreement with IZ was moderate for MRI and fair for PET (table 3).

Concordance with SOZ for the EMSI methods was between $35 \%$ and $73 \%$ (table 2). For ECD, this was higher in BESA than in CURRY both for EEG $(p=0.003)$ and MEG $(p=$ 0.002 ). For DSM, there was no difference between the 2 software packages. Concordance with SOZ for CURRY DSM cEMSI and CURRY ECD MEG was significantly higher than for MRI ( $p=0.036$ and $p=0.029$, respectively) (data available from Dryad, appendix 6, doi.org/10.5061/dryad.p4r01pq). Concordance with SOZ for CURRY ECD MEG was higher than for PET $(p=0.033)$. Concordance with SOZ for the

Figure 4 Clinical utility of EMSI

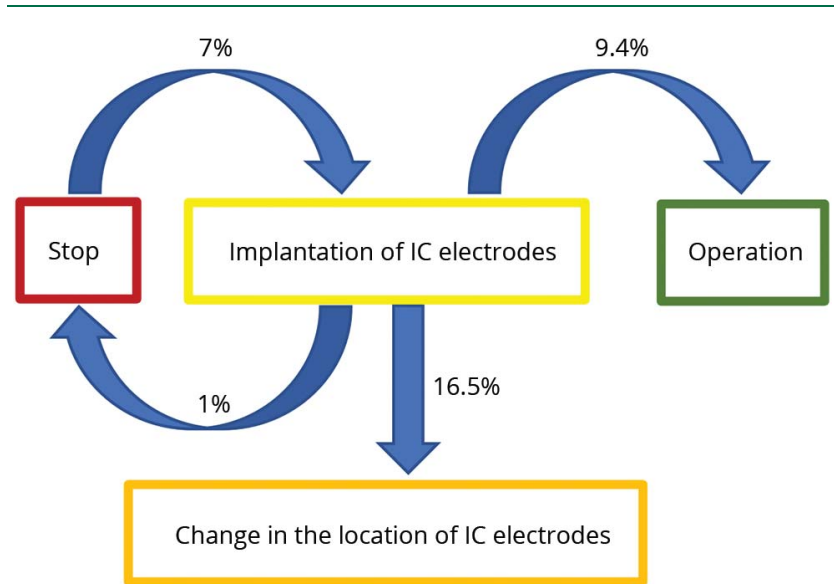

In $34 \%$ of patients $(29 / 85)$, EMSI changed the management plan. The changes were distributed as follows: stop $\rightarrow$ implantation of intracranial electrodes, $6 / 85$ (7\%); implantation $\rightarrow$ stop $1 / 85(1 \%)$, change in the location of implanted electrodes, $14 / 85(16.5 \%)$; skipping implantation and going directly to operation, 8/85 (9.4\%). EMSI = electromagnetic source imaging; IC = intracranial electrodes. 
Table 2 Performance of electromagnetic source imaging, MRI, and PET in presurgical evaluation

\begin{tabular}{|c|c|c|c|c|c|c|c|c|}
\hline \multirow{2}{*}{$\frac{\text { Reference standard }}{\text { Outcome measure }}$} & \multicolumn{2}{|l|}{ ICR } & \multicolumn{6}{|c|}{ Seizure-free patients 1 y after operation } \\
\hline & Concordance with $\mathrm{IZ}, \%$ & Concordance with SOZ, \% & Sensitivity, \% & Specificity, \% & PPV, \% & NPV, \% & Localization accuracy, \% & Odds ratio \\
\hline CURRY ECD EEG & $56(37-74)$ & $35(14-56)$ & $10(0-22)$ & $90(78-100)$ & $60(17-100)$ & $42(28-57)$ & $44(30-58)$ & $1.3(0.2-7.2)$ \\
\hline CURRY DSM EEG & $73(56-90)$ & $50(28-72)$ & $17(3-31)$ & $90(78-100)$ & $71(38-100)$ & $44(29-59)$ & $48(34-62)$ & $0.8(0.1-5.6)$ \\
\hline CURRY ECD MEG & $65(48-81)$ & $35(15-55)$ & $33(16-50)$ & $90(78-100)$ & $83(62-100)$ & $49(33-65)$ & $57(43-71)$ & $4.7(0.7-30.8)$ \\
\hline CURRY DSM MEG & $84(72-96)$ & $67(48-86)$ & $43(25-61)$ & $76(58-95)$ & $72(51-93)$ & $48(31-66)$ & $57(43-71)$ & $2.0(0.4-10.3)$ \\
\hline CURRY ECD CEMSI & $53(31-71)$ & $36(16-67)$ & $31(14-48)$ & $81(64-98)$ & $69(44-95)$ & $46(30-62)$ & $52(38-66)$ & $5.8(0.9-36.4)$ \\
\hline CURRY DSM CEMSI & $80(66-94)$ & $64(43-84)$ & $38(20-56)$ & $76(58-95)$ & $69(46-92)$ & $47(30-64)$ & $54(40-68)$ & $19.2(1.8-204.7)$ \\
\hline BESA ECD EEG & 87 (75-99) & $73(54-92)$ & $31(14-48)$ & $71(52-91)$ & $60(35-85)$ & $43(26-59)$ & $48(34-62)$ & $1.0(0.2-5.2)$ \\
\hline BESA DSM EEG & $74(59-90)$ & $64(43-84)$ & $34(17-52)$ & $71(52-91)$ & $63(39-86)$ & $44(27-61)$ & $50(36-64)$ & $0.5(0.1-3.2)$ \\
\hline BESA ECD MEG & 89 (78-99) & $65(45-85)$ & $43(25-61)$ & 76 (58-95) & $72(51-93)$ & $48(31-66)$ & $57(43-71)$ & $3.0(0.6-15.1)$ \\
\hline BESA DSM MEG & $82(69-95)$ & $63(43-82)$ & $43(25-61)$ & $76(58-95)$ & $72(51-93)$ & $48(31-66)$ & $57(43-71)$ & $3.6(0.7-17.4)$ \\
\hline MRI & $50(31-69)$ & $37(15-89)$ & $67(50-84)$ & $57(36-79)$ & $69(52-86)$ & $55(34-76)$ & $63(49-76)$ & - \\
\hline PET & $29(13-45)$ & $39(16-62)$ & $59(35-82)$ & $81(62-100)$ & 77 (54-100) & $65(44-86)$ & $70(54-86)$ & - \\
\hline
\end{tabular}

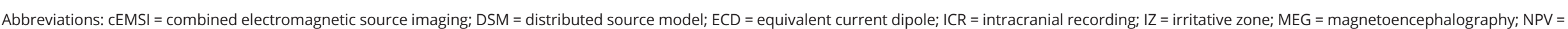
negative predictive value; PPV = positive predictive value; $\mathrm{SOZ}$ = seizure onset zone.

Data represent proportion (95\% confidence interval). Since none of the seizure-free patients were operated discordant to MRI or PET, odds ratio could not be determined for these methods. 
Table 3 Agreement between imaging methods and intracranial recording

\begin{tabular}{lll}
\hline & Agreement with IZ & Agreement with SOZ \\
\hline CURRY ECD CEMSI & $0.58(0.38-0.78)$ & $0.38(0.20-0.58)$ \\
\hline CURRY DSM CEMSI & $0.56(0.38-0.73)$ & $0.40(0.22-0.58)$ \\
\hline CURRY ECD MEG & $0.49(0.32-0.67)$ & $0.33(0.17-0.50)$ \\
\hline CURRY DSM MEG & $0.60(0.43-0.77)$ & $0.39(0.24-0.55)$ \\
\hline CURRY ECD EEG & $0.58(0.38-0.77)$ & $0.27(0.10-0.45)$ \\
\hline CURRY DSM EEG & $0.56(0.38-0.73)$ & $0.30(0.14-0.49)$ \\
\hline BESA ECD MEG & $0.63(0.47-0.80)$ & $0.42(0.24-0.58)$ \\
\hline BESA DSM MEG & $0.61(0.45-0.78)$ & $0.41(0.25-0.60)$ \\
\hline BESA ECD EEG & $0.71(0.52-0.87)$ & $0.33(0.16-0.51)$ \\
\hline BESA DSM EEG & $0.71(0.51-0.88)$ & $0.34(0.15-0.55)$ \\
\hline MRI & $0.41(0.13-0.69)$ & $0.32(0.06-0.56)$ \\
\hline PET & $0.32(0.11-0.57)$ & $0.32(0.12-0.57)$ \\
\hline
\end{tabular}

Abbreviations: $\mathrm{CEMSI}=$ combined electromagnetic source imaging; DSM = distributed source model; $E C D$ = equivalent current dipole; $I Z$ = irritative zone; $\mathrm{MEG}$ = magnetoencephalography; $\mathrm{SOZ}$ = seizure onset zone. Data represent $\mathrm{k}$ values ( $95 \%$ confidence intervals).

remaining EMSI methods did not differ from PET (data available from Dryad, appendix 7). Agreement between $\mathrm{SOZ}$ and DSM cEMSI in CURRY and between SOZ and both MSI methods in BESA (ECD and DSM) was moderate. The remaining EMSI analysis, MRI, and PET had a fair agreement with SOZ (table 3).

\section{Operated patients}

Of the 57 patients who had resective surgery, 51 patients (27 males; age: 8-62, median 62 years) were operated more than 1 year ago (figure 3 ). Thirty-one patients had temporal foci and 20 had extratemporal foci (frontal 15, parietal 3, occipital 2 ). The median time from MEG-EEG recording to operation was 10 months (range: 1-48 months). Thirty patients (59\%) were seizure-free at 1-year postoperative follow-up (figure 3).

Table 2 summarizes the measures determined from the operation outcome: sensitivity, specificity, PPV, NPV, and localization accuracy. See data available from Dryad, appendices 6 and 7, doi.org/10.5061/dryad.p4r01pq.

Localization accuracy (proportion of TPs and TNs) of EMSI methods was between $44 \%$ and $57 \%$. There was no significant difference in localization accuracy among the EMSI methods and between the EMSI methods and MRI. There was no significant difference between the localization accuracy of PET and EMSI in BESA and cEMSI in CURRY.

Sensitivity of EMSI methods was between $10 \%$ and $43 \%$ (table 2). For ESI using ECD, sensitivity was significantly higher in BESA than in CURRY $(p=0.02)$. There was no significant difference in sensitivity among the other EMSI methods. Sensitivity of MRI (67\%; 50\%-84\%) was significantly higher than most EMSI methods, except for MSI (ECD and DSM) using BESA and DSM of MEG-EEG signals using CURRY. There was no significant difference in sensitivity between PET (59\%; 35\%-82\%) and DSMs (cEMSI in CURRY, MEG in CURRY and BESA, EEG in BESA) and between PET and ECD ESI in BESA. PET had significantly higher sensitivity than the other EMSI methods. See data available from Dryad, appendices 6 and 7, doi.org/10.5061/ dryad.p4r01pq.

Specificity of EMSI methods was between $71 \%$ and $90 \%$ (table 2). ESI in BESA had significantly higher specificity than using CURRY. Specificity was higher for all EMSI methods than MRI (57\%; 36\%-79\%), and this was statistically significant for all methods except for cEMSI. There was no significant difference in specificity between EMSI methods and PET. See data available from Dryad, appendices 6 and 7, doi. org/10.5061/dryad.p4r01pq.

EMSI had PPV between $60 \%$ and $83 \%$ and NPV between $42 \%$ and $49 \%$ (table 2). There was no significant difference in PPV among the EMSI methods and between the EMSI methods and conventional neuroimaging methods (MRI and PET). In addition, there was no significant difference between NPV of MRI and EMSI. NPV of PET was higher than that of EMSI. This was significant compared to ESI and MSI in CURRY, the cEMSI analysis (ECD) in CURRY, and for the analysis of EEG using ECD in BESA. See data available from Dryad, appendices 6 and 7, doi.org/10.5061/ dryad.p4r01pq.

Since none of the seizure-free patients were operated discordant to MRI or PET, OR could not be determined for these methods. There was no significant difference in OR of ESI and MSI between the 2 software packages. OR for MSI was between 2 and 4.7 and for ESI from 0.5 to 1.25 (data available from Dryad, appendix 8, doi.org/10.5061/dryad. p4r01pq). Combining both EEG and MEG signals (cEMSI) gave an OR of 5.8 for ECD and 19.2 for DSM (table 2). OR of cEMSI using DSM was significantly higher than both ESI ( $p=$ $0.02)$ and MSI $(p=0.03)$.

Additional data with the distribution of the results in different lesion types and locations (temporal vs extratemporal) are available from Dryad (appendix 9, doi.org/10.5061/dryad. p4r01pq).

\section{Discussion}

More than one-fourth (28\%) of the ED clusters were visible in only one modality (MEG: 13\%; EEG: $15 \%$ ). Thus, although most clusters were visible in both modalities, in order to record all clusters, simultaneous MEG and EEG recording is 
needed. EMSI showed focal epileptiform abnormalities localized at sublobar level in $67 \%$ of the patients.

Despite using standardized methodology, variability introduced by different software packages and operators was higher than the variability introduced by using different inverse solutions (ECD vs DSM) by the same operator, in the same software. This calls for further standardization.

EMSI methods achieved substantial agreement with IZ, but only moderate with SOZ (MSI and cEMSI). This is not surprising, since EMSI analyzed interictal EDs, which is the IZ. Although IZ is often in the same region as SOZ, ICR clearly showed ${ }^{24}$ that this is not always the case. Previous studies suggested that the "dominant" cluster of interictal discharges is concordant with the SOZ. ${ }^{7}$ However, these studies did not define what was "dominant." Although we have prospectively defined what we considered dominant clusters, we found that source imaging of interictal discharges correlated better with IZ than with SOZ.

Using postoperative outcome as reference standard, we found that the accuracy of EMSI was not significantly different from MRI (none of the EMSI methods) and from PET (MSI in BESA and cEMSI in CURRY). Although sensitivity of MRI was higher than most EMSI methods, its specificity was lower than EMSI. There was no significant difference in sensitivity and specificity between most EMSI methods and PET. Using DSM for analyzing the combined dataset of MEG and EEG yielded significantly higher OR than separate analysis of both datasets. This further emphasized the clinical importance of recording MEG and EEG simultaneously.

The accuracy of EMSI was relatively low, though similar to the conventional neuroimaging methods. Localizing the epileptogenic zone is extremely difficult, and there is no single method that on its own is sufficient for the presurgical evaluation. Although in the whole group of patients, the accuracy of EMSI was not significantly different from conventional neuroimaging, these methods complemented each other, since EMSI was able to localize the source in cases in which the conventional methods did not: EMSI provided new information that changed patients' management plan in onethird of the individual cases. In $80 \%$, these changes proved to be useful at 1-year follow-up. This demonstrates the role of EMSI in the multimodal presurgical evaluation of patients with drug-resistant focal epilepsy.

This study has several limitations. Almost all patients in whom EMSI was part of the clinical workup consented to the study (normal MRI or discordant MRI, EEG, and semiology). However, this was not the case for patients whose decision about operation was reached based on concordant MRI, EEG, and semiology and where EMSI was not part of the clinical workup. Thus, more complex cases might be overrepresented in our study.
All patients were undergoing a presurgical evaluation in the Danish national epilepsy surgery program, and all MEG-EEG were recorded and analyzed at Aarhus University Hospital. Although we addressed the variability introduced by 2 different software packages and analyzers, our study is singlecenter.

Perfect reference standard lacks for presurgical evaluation. ${ }^{6}$ Because of spatial sampling problems, ICRs can be misleading. However, using resection site and postoperative outcome as reference standard has its drawbacks too: despite correct localization of IZ and SOZ, patients might not become seizure-free, since IZ and SOZ do not necessarily coincide with the epileptogenic zone. Therefore, we opted for using both datasets as reference standard, and here we emphasize the intrinsic limitations of both approaches.

Our high-density EEG array recorded simultaneously with the MEG was between 64 and 80 electrodes. Although some studies suggested that at least 128 electrodes are needed for optimal ESI, 7 other studies failed to find increase in accuracy beyond 64 electrodes. ${ }^{25}$ However, not only the number of electrodes matters, but also their distribution (even spatial sampling) and coverage of inferior parts of the head (cheek and neck). Our electrode array was in accordance with these principles.

Previous studies using simulated data, somatosensory responses and a case study, emphasized the importance of individual realistic conductivity estimations for combined EEG and MEG source analysis. ${ }^{17,26,27}$ Because of the complementary nature of the 2 modalities and the increased number of sensors, superior spatial resolution was achieved. This is in line with our findings that the OR was highest for cEMSI.

The evidence provided by this study is classified as Class IV, because implantation of intracranial electrodes was not blinded to the results of the EMSI and $<80 \%$ of the patients had been implanted or operated. Since locations shown by EMSI need to be implanted for validation, it is technically impossible to do this blinded to the EMSI. Furthermore, typically less than half of the patients entering presurgical evaluation are implanted or operated. Therefore, it is practically impossible to achieve a higher class of evidence for diagnostic studies in presurgical evaluation of patients with epilepsy.

Our results indicate that EMSI is as accurate as the wellestablished neuroimaging methods and it provides clinically useful, nonredundant information for the presurgical evaluation of patients with epilepsy.

\section{Author contributions}

L.D., A.F.-F., and S.B. contributed to the conception and design of the study. L.D., S.B., P.O.H., P.S., A.S., L.H.P., M.F., G. Rásonyi, G. Rubboli, B.P., A.-M.L., O.M.H., P.U., B.O., and J.B. acquired and analyzed data. L.D. and S.B. drafted the 
manuscript and the figures. All authors contributed to editing the final manuscript.

\section{Acknowledgment}

The authors express their gratitude to neurophysiology technicians Henrik Søvsø and Esben Holch Porsborg (Aarhus University Hospital) for the MEG-EEG recordings, to Christopher Bailey (Aarhus University) for technical assistance with the MEG recordings, to Michael Scherg (BESA) and Jörn Kastner (Compumedics) for assistance with development of the standardized analysis pipeline, and to Aparna Udupi (Aarhus University) for help with the statistical analysis. Per Sidenius is a deceased author.

\section{Study funding}

Supported by Aarhus University, Lundbeck Foundation, The Velux Foundations, the Danish Epilepsy Association, Helene and Viggo Bruuns Fund, and Lennart Grams Memorial Fund.

\section{Disclosure}

L. Duez, H. Tankisi, and P. Hansen report no disclosures relevant to the manuscript. P. Sidenius is deceased; disclosures are not included for this author. A. Sabers, L. Pinborg, M. Fabricius, G. Rásonyi, G. Rubboli, B. Pedersen, A. Leffers, P. Uldall, B. Jespersen, J. Brennum, O. Henriksen, and A. Fuglsang-Frederiksen report no disclosures relevant to the manuscript. S. Beniczky reports nonfinancial support from Elekta, personal fees from Sage Therapeutics, Brain Sentinel, Eisai, and UCB, outside the submitted work. Go to Neurology.org/N for full disclosures.

\section{Publication history}

Received by Neurology April 11, 2018. Accepted in final form October 2, 2018.

\section{References}

1. Sander JW. The epidemiology of epilepsy revisited. Curr Opin Neurol 2003;16: $165-170$.

2. Kwan P, Brodie MJ. Early identification of refractory epilepsy. N Engl J Med 2000; 342:314-319.

3. Rosenow F, Luders H. Presurgical evaluation of epilepsy. Brain 2001;124:1683-1700.

4. Mouthaan BE, Rados M, Barsi P, et al. Current use of imaging and electromagnetic source localization procedures in epilepsy surgery centers across Europe. Epilepsia 2016;57:770-776.
5. Knowlton RC, Razdan SN, Limdi N, et al. Effect of epilepsy magnetic source imaging on intracranial electrode placement. Ann Neurol 2009;65:716-723.

6. De Tiege X, Carrette E, Legros B, et al. Clinical added value of magnetic source imaging in the presurgical evaluation of refractory focal epilepsy. J Neurol Neurosurg Psychiatry 2012;83:417-423.

7. Brodbeck V, Spinelli L, Lascano AM, et al. Electroencephalographic source imaging a prospective study of 152 operated epileptic patients. Brain 2011;134:2887-2897.

8. Beniczky S, Lantz G, Rosenzweig I, et al. Source localization of rhythmic ictal EEG activity: a study of diagnostic accuracy following STARD criteria. Epilepsia 2013;54: 1743-1752.

9. Bossuyt PM, Cohen JF, Gatsonis CA, Korevaar DA; STARD Group. STARD 2015: updated reporting guidelines for all diagnostic accuracy studies. Ann Transl Med 2016;4:85.

10. Lantz G, Spinelli L, Seeck M, De Peralta Menendez RG, Sottas CC, Michel CM. Propagation of interictal epileptiform activity can lead to erroneous source localizations: a 128-channel EEG mapping study. J Clin Neurophysiol 2003;20: 311-319.

11. Almubarak S, Alexopoulos A, Von-Podewils F, et al. The correlation of magnetoencephalography to intracranial EEG in localizing the epileptogenic zone: a study of the surgical resection outcome. Epilepsy Res 2014;108:1581-1590.

12. Taulu S, Kajola M, Simola J. Suppression of interference and artifacts by the signal space separation method. Brain Topogr 2004;16:269-275.

13. Bagic AI, Knowlton RC, Rose DF, Ebersole JS. American Clinical Magnetoencephalography Society Clinical Practice Guideline 1: recording and analysis of spontaneous cerebral activity. J Clin Neurophysiol 2011;28:348-354.

14. Birot G, Spinelli L, Vulliémoz S, et al. Head model and electrical source imaging: a study of 38 epileptic patients. Neuroimage Clin 2014;5:77-83.

15. Beniczky S, Aurlien H, Brogger JC, et al. Standardized Computer-Based Organized Reporting of EEG: SCORE. Epilepsia 2013;54:1112-1124.

16. Wagner M, Fuchs M, Kastner J. Evaluation of sLORETA in the presence of noise and multiple sources. Brain Topogr 2004;16:277-280.

17. Fuchs M, Wagner M, Wischmann HA, et al. Improving source reconstructions by combining bioelectric and biomagnetic data. Electroencephalogr Clin Neurophysiol 1998;107:93-111.

18. Fuchs M, Kastner J, Wagner M, Hawes S, Ebersole JS. A standardized boundary element method volume conductor model. Clin Neurophysiol 2002;113:702-712.

19. Seeck M, Koessler L, Bast T, et al. The standardized EEG electrode array of the IFCN Clin Neurophysiol 2017;128:2070-2077.

20. Gaspard N, Hirsch LJ, LaRoche SM, Hahn CD, Brandon M. Interrater agreement for critical care EEG terminology. Epilepsia 2014;55:1366-1373.

21. Landis JR, Koch GG. The measurement of observer agreement for categorical data. Biometrics 1977;33:159-174.

22. McCullagh P, Nelder JA. Generalized Linear Models, 2nd ed. London: Chapman \& Hall; 1989.

23. Agresti A. Categorical Data Analysis, 2nd ed. Hoboken, NJ: John Wiley \& Sons; 2002: $70-114$.

24. Fisher RS, Scharfman HE, deCurtis M. How can we identify ictal and interictal abnormal activity? Adv Exp Med Biol 2014;813:3-23.

25. Lantz G, Grave de Peralta R, Spinelli L, Seeck M, Michel CM. Epileptic source localization with high density EEG: how many electrodes are needed? Clin Neurophysiol 2003;114:63-69.

26. Huang MX, Song T, Hagler DJ Jr, et al. A novel integrated MEG and EEG analysis method for dipolar sources. Neuroimage 2007;37:731-748.

27. Aydin Ü, Vorwerk J, Küpper P, et al. Combining EEG and MEG for the reconstruction of epileptic activity using a calibrated realistic volume conductor model. PLoS One 2014;9:e93154.

28. Bast T, Oezkan O, Rona S, et al. EEG and MEG source analysis of single and averaged interictal spikes reveals intrinsic epileptogenicity in focal cortical dysplasia. Epilepsia 2004;45:621-631. 


\section{Neurology}

\section{Electromagnetic source imaging in presurgical workup of patients with epilepsy: A prospective study}

Lene Duez, Hatice Tankisi, Peter Orm Hansen, et al.

Neurology 2019;92;e576-e586 Published Online before print January 4, 2019

DOI 10.1212/WNL.0000000000006877

This information is current as of January 4, 2019

\section{Updated Information \&} Services

References

Citations

Subspecialty Collections

Permissions \& Licensing

Reprints including high resolution figures, can be found at: http://n.neurology.org/content/92/6/e576.full

This article cites 26 articles, 1 of which you can access for free at: http://n.neurology.org/content/92/6/e576.full\#ref-list-1

This article has been cited by 1 HighWire-hosted articles: http://n.neurology.org/content/92/6/e576.full\#\#otherarticles

This article, along with others on similar topics, appears in the following collection(s):

\section{Class IV}

http://n.neurology.org/cgi/collection/class_iv

Cortical localization

http://n.neurology.org/cgi/collection/cortical_localization

EEG

http://n.neurology.org/cgi/collection/eeg

Epilepsy surgery

http://n.neurology.org/cgi/collection/epilepsy_surgery_

Functional neuroimaging

http://n.neurology.org/cgi/collection/functional_neuroimaging

Information about reproducing this article in parts (figures,tables) or in its entirety can be found online at:

http://www.neurology.org/about/about_the_journal\#permissions

Information about ordering reprints can be found online:

http://n.neurology.org/subscribers/advertise

Neurology ${ }^{\circledR}$ is the official journal of the American Academy of Neurology. Published continuously since 1951, it is now a weekly with 48 issues per year. Copyright Copyright (C) 2019 The Author(s). Published by Wolters Kluwer Health, Inc. on behalf of the American Academy of Neurology.. All rights reserved. Print ISSN: 0028-3878. Online ISSN: 1526-632X.

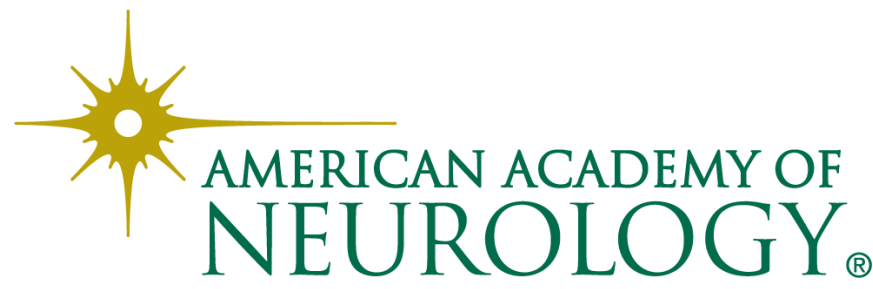

